\title{
Bethe-Salpeter Study of the Optical Absorption of trans and cis Azobenzene-Functionalized Metal-Organic Frameworks using Molecular and Periodic Models
}

\author{
Aseem Rajan Kshirsagar, ${ }^{\dagger}$ Claudio Attaccalite, ${ }^{\ddagger}$ Xavier Blase, ${ }^{\uparrow}$ Jing $\mathrm{Li}^{\S}{ }^{\S}$ and Roberta Poloni*,† \\ $\dagger$ Univ. Grenoble Alpes, CNRS, Grenoble-INP, SIMaP, 38000 Grenoble, France \\ $\ddagger C N R S / A i x$-Marseille Université, Centre Interdisciplinaire de Nanoscience de Marseille UMR 7325, \\ Campus de Luminy, 13288 Marseille Cedex 9, France \\ ฯUniv. Grenoble Alpes, CNRS, Institut Néel, 38042 Grenoble, France \\ $\S$ Univ. Grenoble Alpes, CEA, Leti, 38000 Grenoble, France \\ Received February 16, 2021; E-mail: roberta.poloni@grenoble-inp.fr
}

\begin{abstract}
The optical absorption spectra of the azobenzenefunctionalized metal-organic framework, PCN-123, are calculated in cis and trans configurations using the Bethe-Salpeter equation (BSE) formalism and the $G W$ approximation using periodic and non-periodic models. In the visible, near-UV and mid-UV region the optical excitations in the MOF are associated with the azobenzene functionalities and this results in spectral features similar to the case of the gas phase azobenzene and the azo-functionalized ligand. The most noticeable difference is the significantly more intense $S_{1}$ band for cis in the MOF as compared to the free molecules which points to a faster and more complete cis $\rightarrow$ trans isomerization in the framework, with strong implications for the design of MOFs with high photoconversion efficiencies. Consistent with these findings, all the molecular models employed to represent the MOF, including the smallest, are found to yield a reasonable description of the low energy optical spectra (between 2 and $5 \mathrm{eV}$ ) of the periodic framework, with the exception of the stronger $S_{1}$ band of $c$ is in the MOF, a feature that we attribute to a limitation of the fragment model to correctly represent the wavefunction of the extended framework.
\end{abstract}

\section{Introduction}

Due to their controllable chemical structure and porosity, metal-organic frameworks (MOFs) have attracted much attention during the past 20 years for a wide range of applications such as chemical sensing, ${ }^{1}$ gas storage, ${ }^{2}$ gas separation, ${ }^{3}$ catalysis, ${ }^{4}$ electronics, ${ }^{5}$ optoelectronics,,${ }^{6,7}$ and energy storage devices. ${ }^{8}$ The high internal surface area, pore volume, and selective affinity ${ }^{9,10}$ for $\mathrm{CO}_{2}$ make MOFs among the best candidate materials for $\mathrm{CO}_{2}$ capture. ${ }^{11-15}$ The main issue associated with carbon capture technologies is that the adsorption and desorption cycles, performed for example by using temperature swing adsorption, are associated with large energy penalties and therefore high operating costs. ${ }^{16}$

A fascinating strategy proposed to perform more energyefficient adsorption/desorption cycles is the use of lightresponsive frameworks. In these MOFs, the desorption of certain gases can be obtained using molecular photoswitches. Specifically, the use of light excitation, preferably in the visible range, rather than temperature for example, can enable a more energy efficient separation process, because the use of thermal energy can be fully or partially by-passed in favour of filtered sunlight, thus potentially reducing the cost of the separation/capture process.

The first work showing a reversible change in gas uptake upon light irradiation was reported in 2012 by Park et al. for PCN-123 MOF. ${ }^{17}$ By functionalizing the ligands of MOF- $5^{18}$ with azobenzene moieties, the authors reported a significant change in $\mathrm{CO}_{2}$ uptake upon UV-Vis irradiation possibly as a consequence of the photoisomerization of the azobenzenes anchored to the MOF scaffold. In the following years, a few experimental studies confirmed that the change in the gas adsorption properties in other photoresponsive azobenzene-functionalized MOFs is indeed related to different fractions of cis and trans isomers within the MOF. ${ }^{19-23}$ In the case of PCN-123, the microscopic mechanism behind the reversible adsorption and desorption of $\mathrm{CO}_{2}$ was later identified by some of us to be the blocking and unblocking of the metal node, i.e. the strongest adsorption site for $\mathrm{CO}_{2}$, by cis and trans configurations, respectively. ${ }^{24}$ Larger photoisomerization yields were predicted to result in larger differences in gas uptake ${ }^{24}$ between adsorption and desorption thus contributing to enhance the efficiency of the process. As such, designing MOFs with more efficient photoswitching properties can be considered as one of the central focuses in this field.

In the case of solvated azobenzene molecules, large photoswitching yields are obtained by exploiting the selective absorption of light by cis or trans at specific wavelengths. ${ }^{25-28}$ Typically, cis $\rightarrow$ trans photo-switching is achieved upon irradiation with visible light $(\lambda \sim 420 \mathrm{~nm})$ by exciting the $\mathrm{S}_{1}$ peak (i.e. the $n \rightarrow \pi^{*}$ transition) where cis has a larger molar absorption than trans, and viceversa, the trans $\rightarrow$ cis photo-switching is performed by exciting with UV light $(\lambda \sim 325 \mathrm{~nm})$ the intense $\mathrm{S}_{2}\left(\pi \rightarrow \pi^{*}\right)$ of trans. ${ }^{26}$ In the case of MOFs, a few experimental studies have shown that the main spectral features of functionalized photoresponsive MOFs resemble the ligand case in the UV-Vis region and that photoisomerization does occur in the MOF. ${ }^{19,20,23,29,30}$ However, due to the lack of computational studies on the subject, the nature of the excitations in the MOF and their similarity with the molecular case have not been established yet. This represents the first open question that we want to address in this work. By studying to which extent the optical properties of the functionalizing gas phase molecule or the ligand resemble the periodic MOF we may validate the use of small MOF fragments for future studies. Besides, the resemblance of the character of the excitations between the MOF and the molecular counterpart (azobenzene here) has strong implications on the mechanism of isomerization 
and thus the quantum yield of isomerization.

Despite MOFs being crystalline solids, their electronic bands often feature small or negligible dispersion. ${ }^{31}$ This has motivated several authors to employ molecular fragment models to study the electronic and optical properties of MOF crystals. ${ }^{32-40}$ Using fragments of MOFs, if justified, can be advantageous as it permits to employ accurate wavefunction methods such as coupled-cluster, configuration-interaction, or multi-configurational methods with reasonable computational expense. The second aspect that we want to address is the accuracy of the fragment approach to study the optical absorption properties of this class of materials and this is done here for the paradigmadic case of PCN-123. We employ the Bethe-Salpeter equation (BSE) formalism ${ }^{41-50}$ to describe the two-body electron-hole bound state and the $G W$ approach ${ }^{44,47,48,51-53}$ to compute quasiparticle energies. Compared with the more standard time-dependent density-functional theory ${ }^{54}$ (TD-DFT), the BSE/ $G W$ formalism exhibits a few advantages. The $G W$ eigenvalues used for constructing BSE Hamiltonian are more accurate than DFT Kohn-Sham (KS) eigenvalues. Then, the nonlocal screened Coulomb interaction, $W$, provides an accurate description of the non-local Coulomb interaction between the electron and hole, of importance for charge-transfer or Rydberg excitations. ${ }^{55}$ The latter of the two is possibly the reason behind the success of BSE in accurately describing excitations of different types. ${ }^{56-58}$

First, we assess the validity of a molecular model by employing three different choices of fragments with a progressively increasing number of metal nodes and azobenzene functional groups, and we compare the results with the corresponding fully-functionalized periodic MOF. A comparison is performed at the DFT level using PerdewBurke-Ernzerhof (PBE) parametrized semilocal exchangecorrelation (XC) functional, ${ }^{59}$ at the $G W$ quasiparticle level, and in fine using BSE to study the optical spectra and the character of the excitons. We find that the BSE/ $G W$ spectra of cis and trans PCN-123 closely resemble those of the free azobenzene molecule. The BSE/ $G W / \mathrm{PBE}$ results show that all fragments, including small ones, yield optical spectra in good agreement with the periodic result between 2 and $5 \mathrm{eV}(\sim 250-600 \mathrm{~nm})$, i.e. the region where absorption is dominated by the azobenzene functionalities. Fragment models are used also to compute the cis and trans spectra using BSE/ $G W / \mathrm{PBEh}$, where the PBEh is the PBE global hybrid functional and the amount of exact exchange (EXX) in $\mathrm{PBEh}^{60}$ is tuned have a DFT starting point fulfilling the ionization potential (IP) condition. ${ }^{61}$ This choice is motivated by our recent study ${ }^{62}$ where we showed that this allows to achieve a good quantitative agreement with experimental $\mathrm{S}_{1}$ for a series of solvated azobenzene derivatives. Besides providing the spectral region where the largest selective absorption of each isomer occurs, the calculated spectra point to the possibility of a more efficient cis $\rightarrow$ trans back isomerization in the MOF due to the significantly more intense $\mathrm{S}_{1}$ (i.e. $n \rightarrow \pi^{*}$ ) of cis azobenzene in the framework, as compared to gas phase of solvated azobenzene.

\section{Computational methods}

The $G W$ method is adopted to correct the KS eigenvalues by using a self-consistent procedure on the eigenvalues ${ }^{63,64}$ described below. The KS eigenvalues are corrected pertur- batively, by replacing the exchange-correlation contribution to the KS eigenvalues by the expectation value of $G W$ selfenergy operator $\Sigma\left(r, r^{\prime}, E\right)$ onto the KS eigenstates, as follows:

$$
\epsilon_{n}^{G W}=\epsilon_{n}^{\mathrm{KS}}+\left\langle\phi_{n}^{\mathrm{KS}}\left|\Sigma^{G W}\left(\epsilon_{n}^{G W}\right)-V^{X C}\right| \phi_{n}^{\mathrm{KS}}\right\rangle,
$$

Here, the subscript $n$ corresponds to $n^{\text {th }} \mathrm{KS}$ state, the superscripts KS and $G W$ denote the level of theory, $\left|\phi_{n}^{\mathrm{KS}}\right\rangle$ is the $n^{\text {th }} \mathrm{KS}$ eigenstate, and $V^{X C}$ is the DFT XC potential. For the first iteration, $G_{0} W_{0}$, the $\Sigma\left(r, r^{\prime}, E\right)$ is computed using the input DFT eigenstates $\epsilon_{n}^{\mathrm{KS}}, \phi_{n}^{\mathrm{KS}}$. In the subsequent iteration, the $\epsilon_{n}^{G_{0} W_{0}}$ replace $\epsilon_{n}^{\mathrm{KS}}$ and so forth until the quasiparticle gap is converged within $0.1 \mathrm{eV}$ to yield the socalled evGW quasiparticle energies. Only a limited number of frontier eigenvalues are corrected explicitly at $G W$ level and the rest are corrected using the scissor operator. See below for more details.

The evGW energies and the KS eigenvectors $\epsilon_{n}^{e v G W}, \phi_{n}^{\mathrm{KS}}$ are then used to build the BSE Hamiltonian. Within the BSE formalism, the optical excitations are computed by solving a linear algebra problem similar to the Casida equation of TD-DFT. ${ }^{54}$ The BSE eigenvalue problem can be written compactly as:

$$
\left[\begin{array}{cc}
R & C \\
-C^{*} & -R^{*}
\end{array}\right]\left[\begin{array}{c}
X_{\mu} \\
Y_{\mu}
\end{array}\right]=\Omega_{\mu}\left[\begin{array}{c}
X_{\mu} \\
Y_{\mu}
\end{array}\right]
$$

where $R\left(R^{*}\right)$ represents the resonant coupling between electron-hole excitations (de-excitations), whereas, the offdiagonal blocks $C$ and $C^{*}$ describe the non-resonant coupling between excitations and de-excitations. $X_{\mu}$ and $Y_{\mu}$ are the excitation and de-excitation components, respectively, of the two-body electron-hole $(e h)$ eigenstate $\Psi_{\mu}\left(r_{e}, r_{h}\right)$ expressed in the transition basis $\left[\phi_{v}\left(r_{h}\right) \phi_{c}\left(r_{e}\right)\right]$ and $\mu$ is the index of excitation, with $\phi_{v}$ and $\phi_{c}$ representing the KS valence and conduction states. Thus $\Omega_{\mu}$ are the eigenvalues of BSE, i.e. the excitation energies, and the corresponding eigenvectors $\Psi_{\mu}\left(r_{e}, e_{h}\right)$ are written as:

$$
\Psi_{\mu}\left(r_{e}, r_{h}\right)=\sum_{v c}\left[X_{\mu}^{v c} \phi_{v}\left(r_{h}\right) \phi_{c}\left(r_{e}\right)+Y_{\mu}^{v c} \phi_{c}\left(r_{h}\right) \phi_{v}\left(r_{e}\right)\right]
$$

The eigenvectors yield the probability amplitude of finding the electron and hole at $r_{e}$ and $r_{h}$, respectively. To compare the character of the exciton between the different approaches, we compute and plot for each relevant excitation, $\mu$, the hole-averaged electron density and charge-averaged hole density as follows:

$$
\begin{aligned}
& \rho_{\mu}\left(r_{e}\right)=\int d r_{h} \Psi_{\mu}\left(r_{e}, r_{h}\right) \Psi_{\mu}^{*}\left(r_{e}, r_{h}\right) \\
& \rho_{\mu}\left(r_{h}\right)=\int d r_{e} \Psi_{\mu}\left(r_{e}, r_{h}\right) \Psi_{\mu}^{*}\left(r_{e}, r_{h}\right)
\end{aligned}
$$

\section{Periodic calculations}

The DFT calculations are performed using Quantum ESPRESSO. ${ }^{65,66}$ Optimized norm-conserving Vanderbilt pseudopotentials obtained from PSEUDO-DOJO vault are used to model the interaction between ionic core and the valence electrons. ${ }^{67,68}$ The geometry of PCN-123 MOF was optimized using the rev-vdW-DF2 functional ${ }^{69}$ by taking the global minimum configuration for trans and cis found in our previous work. ${ }^{24}$ Specifically, these geometries are obtained by performing a full geometrical optimization using 

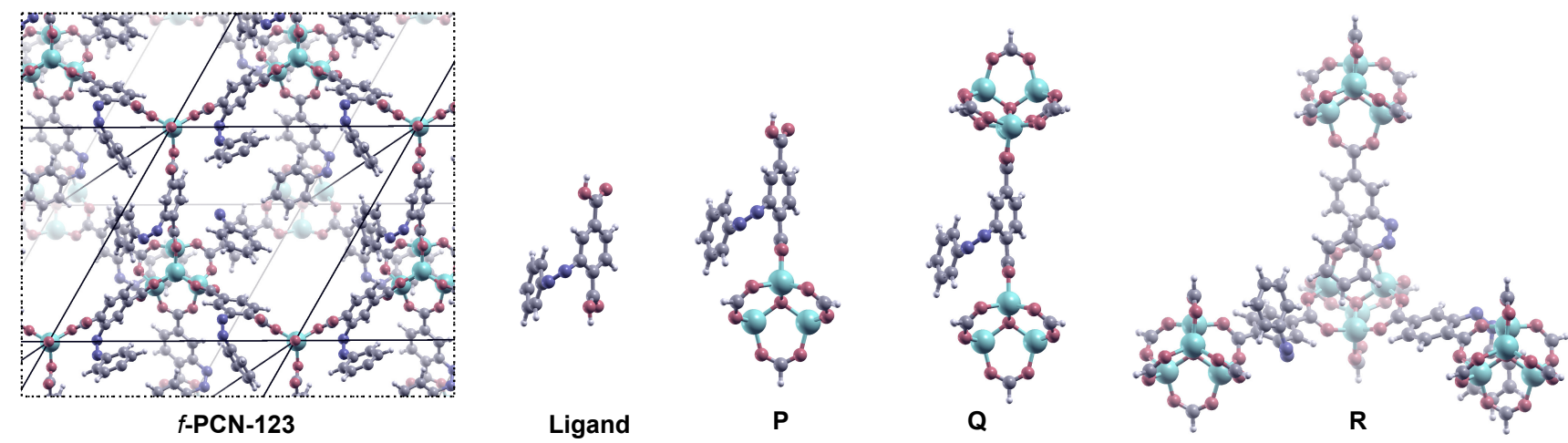

Figure 1. Illustrations of the unit cell of the PCN-123 MOF, the PCN-123 ligand, and fragments $\mathrm{P}$, $\mathrm{Q}$ and $\mathrm{R}$, in cis configuration. Colour code as follows, $\mathrm{C}$ : gray, N: blue, H: white, Zn: cyan, O: red. The periodic MOF is shown by aligning the [111] direction perpendicular to the plane; the black lines outline the boundaries of primitive unit cells of the PCN-123 crystal.

the primitive cell of PCN-123 which contains six ligands and a total of 178 atoms, including the azobenzene functionalities. In cis, all molecular functionalizations where optimized in the cis 2 configuration discussed in Ref. 24, i.e. with each azobenzene ring blocking one metal node. The DFT eigenvalues and eigenstates for excited state calculations are obtained using PBE XC functional. ${ }^{59}$ Kinetic energy cut-off of $80 \mathrm{Ry}$ and $320 \mathrm{Ry}$ is used for truncation of basis set representing Kohn-Sham orbitals and Kohn-Sham charge density respectively. Reciprocal space is sampled using $\Gamma$ point only which is found to be sufficient in absence of band dispersion.

The BSE $/ G W$ calculations are performed using YAMBO. ${ }^{70,71}$ To avoid escalation of computational expenses, the frequency dependence of the dielectric matrix is approximated using Godby-Needs plasmon-pole model. ${ }^{72}$ For the calculation of dielectric screening, a total $3000 \mathrm{KS}$ states are used including the 380 valence states. Out of all the KS states, 128 valence states and 128 conduction states are corrected explicitly by $G W$ and used to build the BSE Hamiltonian. To reduce the cost, only the resonant coupling between the electron and hole is considered for the BSE. For the same reason, the sizes of the plane-waves basis used for different steps in BSE and $G W$ are truncated at lower kinetic energy cut-offs than the cut-off used for DFT. Details of the these truncation parameters and their convergences are provided in Tab. S1 of the supplementary information (SI). Bruneval-Gonze terminator is used to speed-up the convergence of correlation part of the $G W$ self-energy with respect to sum over the number of unoccupied states. ${ }^{73}$

\section{Fragment calculations}

The geometries of the fragments of $\mathrm{PCN}-123$ are constructed by cleaving parts of the fully functionalized (i.e. every ligand is functionalized) and fully optimized PCN-123 $\mathrm{MOF}^{24}$ by terminating unsaturated bonds with $\mathrm{H}$ atoms. Because we focus on the low energy excitations which are possibly associated with the azobenzene functionalization, the fragments are constructed so as to keep the chemical connectivities of the ligand intact. In our earlier work, the structure of the azobenzene functionalization in PCN-123 was found to be sensitive to the MOF milieu due to steric factors and strong van der Waals interactions. ${ }^{24}$ To not deviate from the structure of periodic PCN-123, no further geometrical optimiza- tion is performed on the fragment except for the relaxation of the coordinates of the added $\mathrm{H}$ terminations.

Three different fragment models were adopted and these are shown in Fig. 1. The smallest, named P, includes only one metal node and an azobenzene-functionalized ligand; fragment $\mathrm{Q}$ includes two complete metal nodes and again one functionalized ligand; fragment R (176 atoms), includes four metal nodes and three functionalized ligands. An illustration of the fully functionalized periodic PCN-123, together with the ligand also used for comparison in this work, are also reported in Fig. 1.

The DFT calculations of the fragments are performed using NWChem. ${ }^{74}$ The correlation-consistent basis sets augcc-pVDZ ${ }^{75,76}$ are used for azobenzene, the PCN-123 ligand, and fragments $\mathrm{P}$ and $\mathrm{Q}$. A cheaper minimally-augmented maug-cc-pVDZ ${ }^{77}$ basis set is used for the larger fragment R. LANL2DZ effective core potential (ECP) and the corresponding basis set is used for $\mathrm{Zn}$ in fragment $\mathrm{R} .{ }^{78,79}$ The use of ECP and the smaller basis set for fragment $\mathrm{R}$ causes a blue shift by up to $\sim 0.2 \mathrm{eV}$ in the BSE spectra (see Fig. S1). For fragment $\mathrm{Q}$ and $\mathrm{R}$, both PBE and PBEh with $40 \%$ EXX are used to compute the DFT ground state. Such a percentage of EXX in the PBEh global hybrid functional has been tuned to reproduce the $G W$ quasiparticle ionization potential with the DFT highest-occupied molecular orbital (HOMO) eigenvalue.

The BSE $/ G W$ calculations for fragments are performed using FIESTA. ${ }^{57,63,80}$ Unlike for the periodic case, the frequency dependence of dielectric screening is not approximated by a plasmon-pole function. The contour deformation technique and the Coulomb-fitting resolution of identity (RI-V) with the universal Weigend Coulomb fitting auxiliary basis ${ }^{81}$ are used for integration over frequencies to calculate the $G W$ self-energy $\Sigma\left(r, r^{\prime}, E\right)$. The full BSE Hamiltonian in considered in this case. The number of eigenvalues corrected at $G W$ level and the number of states used to build the BSE Hamiltonian vary for each fragment and they are tabulated in Tab. S2.

To deal with the electrostatic polarization of the fragments due to the MOF environment in the ground state, a conductor like screening model ${ }^{82}$ (COSMO) as implemented in NWChem is used. The MOF environment is thus approximated using the optical dielectric constant of the periodic PCN-123 in the zero-frequency limit $\left(\varepsilon_{\text {opt }} \sim 1.76\right)$ obtained using the random-phase approximation in YAMBO.

To account for the screening by the environment at 
the $G W$ and BSE level, a QM/MM (Quantum Mechanics/Molecular Mechanics) approach is used by a employing discrete polarizable model. ${ }^{83,84}$ The MM part is modeled as a fixed cubic lattice of polarizable pseudo-atoms which are assigned the atomic polarizability computed from the zero-frequency optical dielectric constant $\left(\varepsilon_{o p t}\right.$ or square of the refractive index) of periodic $\mathrm{PCN}-123$ by using the Clausius-Mossotti formula. The reaction-field matrix which describes the screening by the MM part is computed using the MESCAL code. ${ }^{85}$ This screening from the environment is added to the screened Coulomb potential $W$ by solving the following set of equations:

$$
\begin{aligned}
& \tilde{v}=v+v \chi^{M M} v \\
& W=\tilde{v}+\tilde{v} \chi_{0} W
\end{aligned}
$$

where $\chi_{0}$ is the independent-particle susceptibility, $v$ is the bare Coulomb potential which is dressed (i.e. $\tilde{v}$ ) by the environment reaction field, ${ }^{83,84}$ and thus $W$ is the screened Coulomb potential incorporating the screening from the MM part. The $G W$ energies are then corrected by using the $\Delta$ COHSEX correction ${ }^{83}$ (i.e. Coulomb-Hole screened exchange) which is essentially the difference between the $\mathrm{COH}-$ SEX self-energy calculated with and without the MM screening. Practically, first the evGW eigenvalues are calculated in gas phase and then the $\triangle$ COHSEX correction is added. Here, ev $G W$ refers to $G_{3} W_{3}$ and $G_{2} W_{2}$ for PBE and PBEh DFT starting points, respectively. At the first $G W$ step, only the HOMO and LUMO are $G W$ corrected, and the scissor operator is applied. In the subsequent steps, more states are explicitely corrected to yield quasiparticle values converged within $0.1 \mathrm{eV}$ similar to YAMBO. The environmental effect are then included as follows:

$$
\begin{aligned}
\epsilon_{n}^{G W_{e}} & \approx \epsilon_{n}^{G W}+\Delta \epsilon_{n}^{\mathrm{COHSEX}} \\
& =\epsilon_{n}^{G W}+\left\langle\phi_{n}^{K S}\left|\Sigma^{\mathrm{COHSEX} / \mathrm{MM}}-\Sigma^{\mathrm{COHSEX}}\right| \phi_{n}^{K S}\right\rangle
\end{aligned}
$$

A detailed description and the accuracy of use of QM/MM model in conjunction with $G W$ and BSE has been discussed in literature. ${ }^{62,83,84,86,87}$ The screened Coulomb potential including environmental effects is then used for the BSE Hamiltonian. ${ }^{62,87}$

\section{Results and Discussion}

\section{Kohn-Sham ground state}

The density of states (DOS) computed using PBE for the three fragment models of PCN-123, the gas phase azobenzene and the periodic PCN-123, is shown in Fig. 2. We only report the cis case, for clarity, the trans case being similar is reported in Fig. S3. The total DOS (gray) and the projected-DOS (pDOS) are shown in each case. For the latter we plot the DOS projected onto the $2 \mathrm{p}$-states of $\mathrm{N}$ (blue), the $2 \mathrm{p}$-states of the $\mathrm{O}$ atoms connecting the metal-node and the ligand (yellow), and the states associated with the $\mathrm{Zn}_{4} \mathrm{O}$ node (red). The magenta strips in the case of azobenzene highlight the $\pi, n, \pi^{*}$ states which are involved in the excitations found in the UV-Vis region of the absorption spectra and leading to photoisomerization, ${ }^{26}$ as discussed in the introduction. For the PCN-123 ligand, the frontier orbitals retain a character analogous to the $\pi, n$ and $\pi^{*}$ of cis and trans azobenzene (see Fig. S2 of SI) with some mixing of

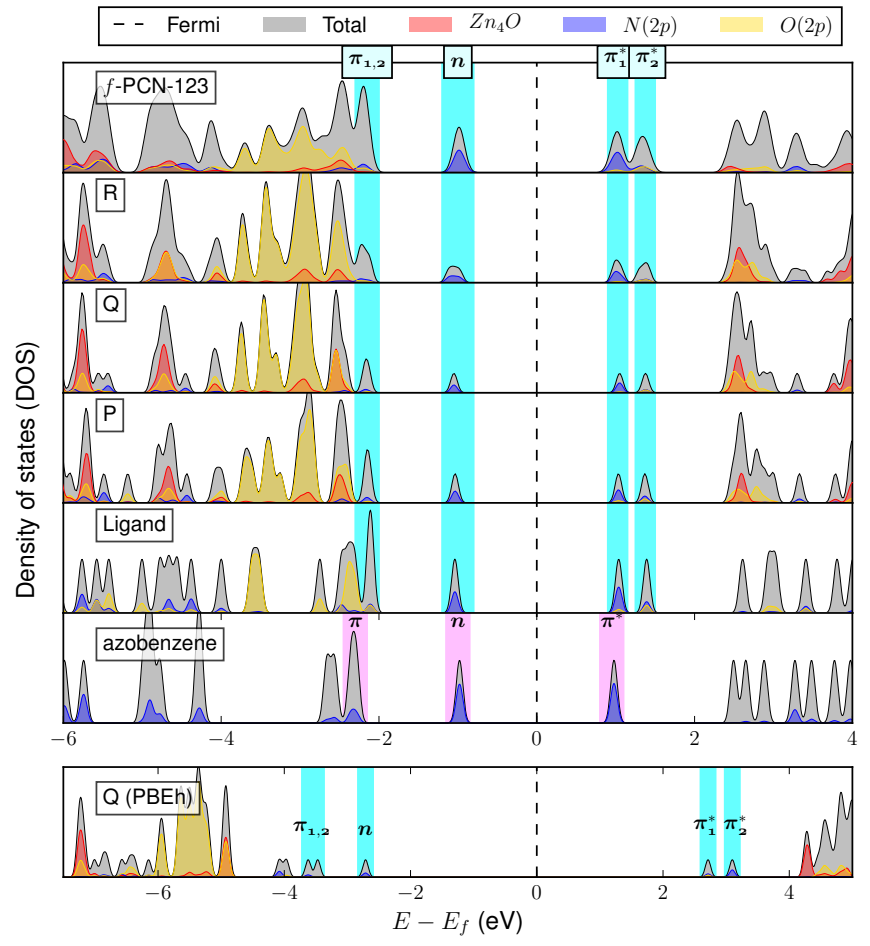

(a)

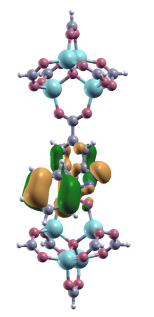

(b) $\pi_{1}$

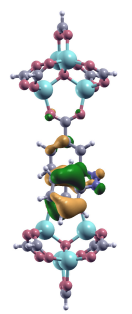

(c) $\pi_{2}$

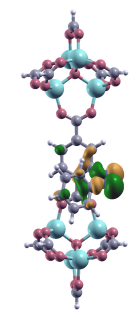

(d) $n$

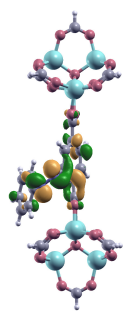

(e) $\pi_{1}^{*}$

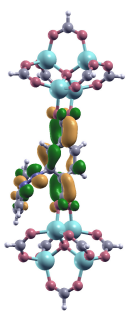

(f) $\pi_{2}^{*}$
Figure 2. (a) pDOS of different models of cis PCN-123 calculated with PBE. The upper panel represents the pDOS of the fully-functionalized periodic system, i.e. $f$-PCN-123 while (P), $(\mathrm{Q})$ and $(\mathrm{R})$ represent fragments with a progressive increase in size as illustrated in Fig. 1. A $0.1 \mathrm{eV}$ broadening is applied in every case. For fragment Q the PBEh (40\% exact exchange) is also plotted for comparison. The DOS and pDOS are presented with different scales for different models. (b) and (c) $\pi$, (d) $n$, (e) $\pi_{1}^{*}$ and (f) $\pi_{2}^{*}$ KS eigenstates of cis fragment Q represented by plotting isosurface of $\left|\phi_{n}\right|^{2}$ with green and yellow depicting opposite signs of the original wavefunction $\pm \phi_{n}$. The midpoint of the fundamental gap is taken as the Fermi level.

p-states of $\mathrm{O}$ atoms into $\pi$ and $\pi^{*}$. Two anti-bonding $\pi$ states close in composition and energy, i.e. $\pi_{1}^{*}$ and $\pi_{2}^{*}$, arise in all cases in place of the single $\pi^{*}$ of azobenzene and are highlighted in cyan in the PDOS. The breaking of symmetry between the two benzene rings upon attachment of the carboxylic acid or groups in one of the two phenyl rings lead to two different $\pi^{*}$ states which localize predominantly on the $\mathrm{N}$ atoms and the benzenedicarboxylic acid (see lower panel of Fig. 2).

The electronic structure of the three fragments, in the vicinity of the band gap, maintains features similar to the periodic MOF in terms of energy and composition (see cyan strips in Fig. 2). The wavefunction corresponding to these states is plotted in the lower panel of Fig. 2 for cis fragment 
Q. A similar behavior is found for all MOF models. For comparison, these states are also plotted for azobenzene and for the ligand in Fig. S2. Even for the ligand, the DOS resembles the MOF case for the KS states near the gap; below $-2.5 \mathrm{eV}$ and above $2.5 \mathrm{eV}$ this agreement vanishes due to the presence of the states associated with the $\mathrm{Zn}_{4} \mathrm{O}$ metal node. Moving away from the gap, the DOS of the three fragments $\mathrm{P}, \mathrm{Q}$ and $\mathrm{R}$ which include single or multiple metaloxide nodes show a reasonable resemblance with the $f$-PCN123 case, for both occupied and unoccupied levels. A similar behavior is found for trans $f$-PCN-123 (see Fig. S3).

The DOS of cis fragment Q calculated using PBEh with $40 \%$ exact exchange is also shown in Fig. 2 for comparison (see Fig. S4 for trans). As expected, the PBE band gap of $2.1 \mathrm{eV}$ for cis and $1.9 \mathrm{eV}$ for trans increases to 5.5 and $5.2 \mathrm{eV}$, respectively, using $\mathrm{PBEh}$, as expected from the EXX-tuning procedure that matches the DFT KS IP onto the evGW level.

Because we do not impose any symmetry constraint during the geometrical optimization, the steric hindrance of the pendant groups inside the MOF results in different configurations of the six azobenzenes within the unit cell and in the twisting and bending of the BDC (benzenedicarboxylate) ligands. ${ }^{24}$ Such geometrical distortions and configurational disorder mimic to some extent the structural disorder of the pendant groups found experimentally in PCN-123 ${ }^{17}$ and lead to the broadening of the DOS and the BSE spectra (vide infra).

\section{The $G W$ correction: fragment ver- sus periodic}

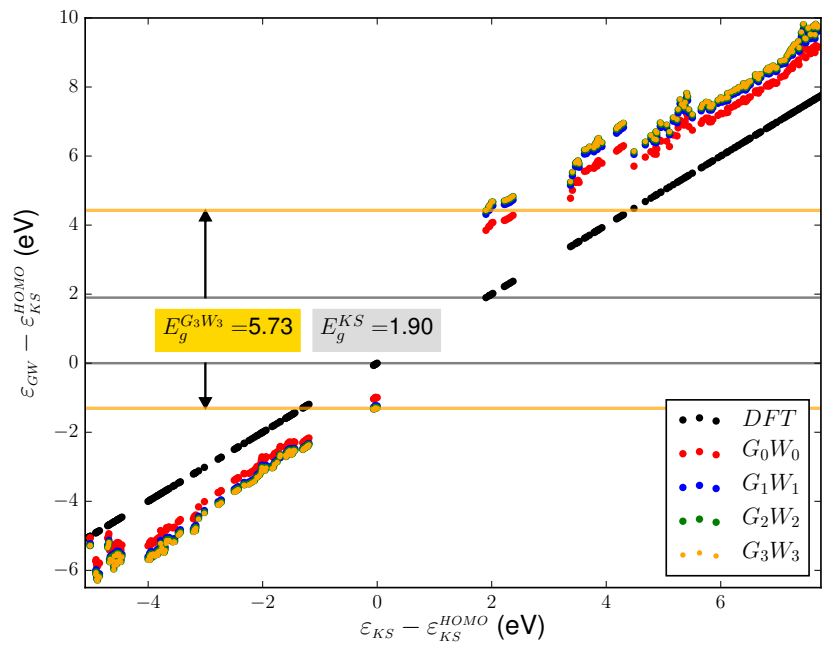

Figure 3. $G W$ quasiparticle energies (in $\mathrm{eV}$ ) of cis $f$-PCN-123 computed at four $G W$ iterations.

The $G W$ quasiparticle energies computed at four $G W$ iterations using YAMBO for the cis $f$-PCN-123 are plotted in Fig. 3. Only the states that are explicitely corrected are shown in the figure. We find that three $G W$ iterations $\left(G_{2} W_{2}\right)$ are sufficient to reach a convergence with criterion of $\Delta E_{g}<0.1 \mathrm{eV}$ (with $E_{g}=$ band gap) with respect to the previous step and the $e v G W$ band gap is $5.73 \mathrm{eV}\left(G_{3} W_{3}\right.$ value), almost $4 \mathrm{eV}$ larger than the PBE gap $(1.9 \mathrm{eV})$. Assuming a similar behaviour of $G W$ convergence for trans $f$-PCN-123, three iterations of $G W$ are performed and deemed sufficient. A similar trend is found with the fundamental gap opening from $1.68 \mathrm{eV}(\mathrm{PBE})$ to $5.31 \mathrm{eV}\left(G_{2} W_{2}\right)$ (see Fig. S5). Henceforth, the term evGW refers to $G_{2} W_{2}$ for YAMBO calculations.

The $G W$ correction was also computed for all the fragment models and for the PCN-123 ligand. The final evGW quasiparticle gaps are reported in Tab. 1. In case of nonperiodic systems, the $e v G W$ eigenvalues are reported after applying the $\triangle$ COHSEX correction accounting for environment screening as explained in the previous section. The $\triangle$ COHSEX procedure closes the band gap by about $\sim 0.7-0.9$ $\mathrm{eV}$ with respect to the gas phase evGW value (see Fig. S9). This arises from polarization (screening) effects that stabilize holes and electrons. The evGW quasiparticle energies for the ligand and fragment models plotted against the KS eigenvalues are reported in Fig. S8.

The comparison of the $e v G W$ quasiparticle corrections computed for the periodic MOF, the three fragment choices, and the ligand, are reported in Fig. S6. Overall, the correction shows the same qualitative behaviour. We find a good quantitative agreement among the non-periodic models, but we note some discrepancy between the non-periodic and the periodic models. The periodic YAMBO calculations yield smaller evGW corrections compared to the non-periodic FIESTA calculations (see Fig. S7). Thus despite the PBE gap being essentially the same for the periodic and non-periodic case (within $0.2 \mathrm{eV}$ ), the periodic ev $G W$ band gap is $\approx 1 \mathrm{eV}$ smaller $(\sim 0.8-1.1 \mathrm{eV})$ compared to the non-periodic case (see Tab. 1). This difference may be attributed to the different computational approaches such as the use of pseudopotentials and the plasmon-pole approximation, in YAMBO, and the use of incomplete bases in FIESTA, as shown and discussed in detail in Ref. 88. Also, on the fragment side, the possible discrepancies on the long-range contribution to the screened Coulomb potential $W$, as described by the QM/MM $\triangle$ COHSEX scheme, may affect the fragment $G W$ calculations. As shown below, the much better agreement of optical BSE excitations, for which the impact of a change in the environmental screening is known to be much smaller, points in this direction. Finally, the fragment approximation may result in somehow different KS eigenstates. This can be inferred by looking at the independent-particle spectra computed for the periodic model and for fragment $\mathrm{Q}$ using the epsilon.x utility of Quantum ESPRESSO (see Fig. S11) for both calculations. Specifically, the ratio between the first two excitations is significantly different in the two cases, with the first excitation being significantly less intense (compared to the second) in the fragment as compared to the periodic MOF.

For non-periodic models, the evGW eigenvalues are calculated also with PBEh with $40 \%$ exact exchange as starting point (see Tab. 1). The hybrid functional used here fulfils the ionization potential theorem such that the DFT HOMO eigenvalue matches with the $e v G W$ ionization potential (within $0.2 \mathrm{eV}$ ). In our earlier work, ${ }^{62}$ we showed that the use of such IP-tuned hybrid functionals to describe the Kohn-Sham ground state yields very accurate BSE/GW excitation energies of crucial $n \rightarrow \pi^{*}$ transition for a range of azobenzene derivatives. The DFT band gap calculated here with PBEh is significantly larger (i.e. $>3 \mathrm{eV}$ ) (see Fig. 2), as expected, compared to the PBE one, bringing it much closer to the evGW band gap. This is further shown in Fig. S10 where the evGW correction is plotted against the 
Table 1. DFT and $e v G W$ gaps for periodic and non-periodic models of PCN-123 calculated with PBE and PBEh (see text). The $e v G W$ gap for the non-periodic calculations are computed by employing an embedding scheme to account for environmental effects at both the ground state and GW level, using $\triangle$ COHSEX for the latter.

\begin{tabular}{ccc|cc|cc|ccc|c}
\hline \hline & \multicolumn{2}{c}{ PBE } & \multicolumn{2}{c}{$\boldsymbol{e v G W} / \mathbf{P B E}$} & \multicolumn{2}{c}{ PBEh } & \multicolumn{2}{c}{$\boldsymbol{e v \boldsymbol { G } \boldsymbol { W } \text { PBEh }}$} \\
\hline \hline Model & cis & trans & cis & trans & cis & trans & cis & trans & code \\
\hline Ligand & 2.08 & 1.94 & 6.59 & 6.44 & 5.39 & 5.24 & 6.71 & 6.53 & FIESTA \\
$\mathbf{P}$ & 2.07 & 1.88 & 6.38 & 6.23 & 5.38 & 5.19 & 6.51 & 6.36 & FIESTA \\
$\mathbf{Q}$ & 2.10 & 1.90 & 6.52 & 6.33 & 5.45 & 5.20 & 6.68 & 6.50 & FIESTA \\
$\mathbf{R}$ & 1.94 & 1.88 & 6.52 & 6.43 & 5.28 & 5.30 & 6.62 & 6.65 & FIESTA \\
\hline $\boldsymbol{f - P C N - 1 2 3}$ & 1.90 & 1.68 & 5.73 & 5.31 & - & - & - & - & YAMBO \\
\hline \hline
\end{tabular}

KS energies for the frontier states of fragment $\mathrm{P}, \mathrm{Q}$ and $\mathrm{R}$ : the $e v G W$ correction is $\sim 1 \mathrm{eV}$ when using the PBEh starting point compared to $\sim 3 \mathrm{eV}$ for $\mathrm{PBE}$. It is interesting to note that, regardless of the XC functional used for the DFT starting point, the $e v G W$ quasiparticle energies converge to close values (see Fig. S12) for both trans and cis, for either the fragments and the ligand, thus confirming the robustness of $G W$ method. ${ }^{89-92}$

\section{The BSE optical spectra: fragment versus periodic}

The BSE/evGW/PBE spectra of cis and trans configurations of $f$-PCN-123 and the fragment models are plotted in Fig. 4. Despite the fact that the difference in the evGW band gap between the periodic and non-periodic calculations reaches up to $0.8 \mathrm{eV}$ for cis and $1.1 \mathrm{eV}$ for trans (see Tab. 1), the energy of the $\mathrm{S}_{1}$ excitation (i.e. $n \rightarrow \pi_{1}^{*}$ ) agrees within $\sim 0.1-0.3 \mathrm{eV}$ for both cis and trans. As mentioned above, this is possibly due to the much reduced impact of environmental screening onto the neutral optical excitations, for which solvatochromic shift are known to be much smaller than the shift in electronic energy levels. As a matter of fact, errors in the screened Coulomb potential enters with opposite sign in the quasiparticle gap than in the electronhole interaction, leading to well-known cancellation of errors at the BSE level. Thus, large differences in the quasiparticle gap may reduce in the corresponding optical gap. ${ }^{93}$

For cis configuration, the first optical band corresponds to the HOMO $\rightarrow$ LUMO $\left(n \rightarrow \pi_{1}^{*}\right)$ transition, similar to the case of gas phase or solvated azobenzene. The second optical band (yellow in Fig. 4), the $\mathrm{S}_{2}$, corresponds to the $n \rightarrow \pi_{2}^{*}$ excitation which is absent in azobenzene. The third (skyblue) optical band, the $\mathrm{S}_{3}$, is due to the $\pi \rightarrow \pi_{1}^{*}$ excitation. The $\mathrm{S}_{4}$, in red, contains mixed $\pi \rightarrow \pi_{1}^{*} / \pi \rightarrow \pi_{2}^{*}$ excitations. From the fourth optical band onwards, the one-to-one correspondence among excitons of different models fades away, mostly owing to the increasing contribution of $\mathrm{O}$ atoms of the $\mathrm{ZnO}_{4}$ noded and to the more delocalized character of the exciton.

For trans, the $\mathrm{S}_{1}$ band (gray) is due to the $n \rightarrow \pi_{1}^{*}$ excitation, whereas the $\mathrm{S}_{2}$ (yellow) is dominated by $\pi \rightarrow \pi_{1}^{*}$ along with some contribution from $n \rightarrow \pi_{2}^{*}$ and $\pi \rightarrow \pi_{2}^{*}$, specifically for fragment $\mathrm{R}$ and the periodic system. The character and energy of $S_{1}$ and $S_{2}$ are similar to the azobenzene molecule. It is important to stress that for fragment $\mathrm{R}$, the energy of $\mathrm{S}_{1}$ and $\mathrm{S}_{2}$ for both cis and trans is blueshifted compared to the smaller PCN-123 fragments possibly due to the use of effective-core-potentials and a smaller basis, as discussed in the computational section. This is shown in Fig. S1 where the effect of these approximations on the calculation of the BSE spectrum is reported for a smaller fragment.

To illustrate the similarities among the different models, the electron-averaged hole density (in yellow) and the holeaveraged electron density (in green) of the $n \rightarrow \pi_{1}^{*}$ and $\pi \rightarrow \pi_{1}^{*}$ transitions are plotted, respectively, for cis and trans, in Fig. 5 for the ligand, fragments $\mathrm{Q}$ and $\mathrm{R}$, and the periodic MOF. For clarity, we neglect fragment $\mathrm{P}$ which exhibits the same character of the exciton. We plot the excitonic density for these two transitions, since these correspond to the most intense bands in the low energy range which are usually employed for excitation in the UV-Vis range to induce large photoisomerization yields. Excitation at the maxima of these two bands will result in efficient trans-to-cis photoisomerization when the $\mathrm{S}_{2}$ (of trans) is excited, and viceversa, when the $\mathrm{S}_{1}$ (of cis) is excited. ${ }^{27,28}$ The excitonic densities of the fragment model are reported in Figs. S14-S18 (for cis) and Figs. S20-S24 (for trans). For the $n \rightarrow \pi_{1}^{*}$ and $\pi \rightarrow \pi_{1}^{*}$ excitations, a good correspondence is found among the different MOF models. The character of the excitation is fairly maintained, however, the exciton may delocalize when models with multiple ligands are employed. In general, as the energy of the exciton increases the character becomes more delocalized (see Figs. S18 and $\mathrm{S} 24$ ). The localization on multiple ligands is found also for certain excitons in the $\mathrm{S}_{1}$ band for both cis (see Figs. S18 and S17) and trans (see Figs. S24 and S23). Furthermore, excitons localized on different ligands can have a different character on each ligand. For example, the exciton number 23 in trans $f$-PCN-123 plotted in Fig. S24 consists of both $n$ and $\pi$ holes on different ligands and, similarly, the electron has both $\pi_{1}^{*}$ and $\pi_{2}^{*}$ character on different ligands.

Overall, the non-periodic models reproduce fairly well the optical absorption of the periodic MOF up to $4.5 \mathrm{eV}$, consistent with excitons being localized on the azobenzenefunctionalized ligands in this spectral region, while at higher energies the absorbance of the $\mathrm{Zn}_{4} \mathrm{O}$ nodes becomes significant. See for example, the excitons of the $\mathrm{S}_{4}$ for cis $f$ PCN-123 (Fig. S18), and the $\mathrm{S}_{3}$ and $\mathrm{S}_{4}$ for trans $f$-PCN123 (Fig. S24). This implies that excitations in the visible, near-UV and mid-UV spectral region can be used to target azobenzene excitation thus possibly resulting in isomerization provided that the excited state dynamics is similar to the molecular case and that steric hindrance is not a limiting factor. This is unlike many other azobenzene based photo-switching systems where use of UV light for azobenzene isomerization is very restricted due to absorption in that range by other chemical entities. ${ }^{94}$

To better visualize the spectral regions with large selective absorption, we plot the $\mathrm{BSE} / \mathrm{e} v G W / \mathrm{PBE}$ spectra of 


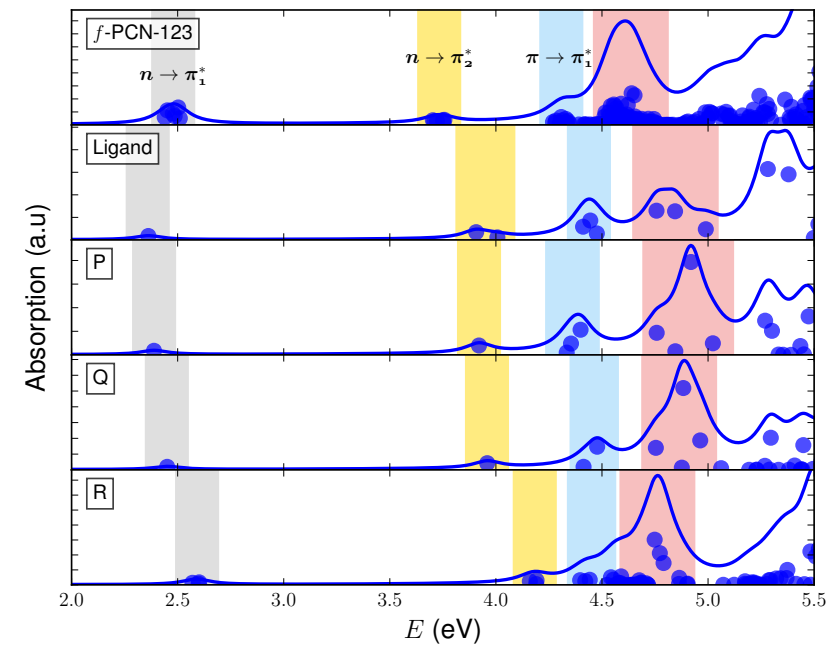

(a)

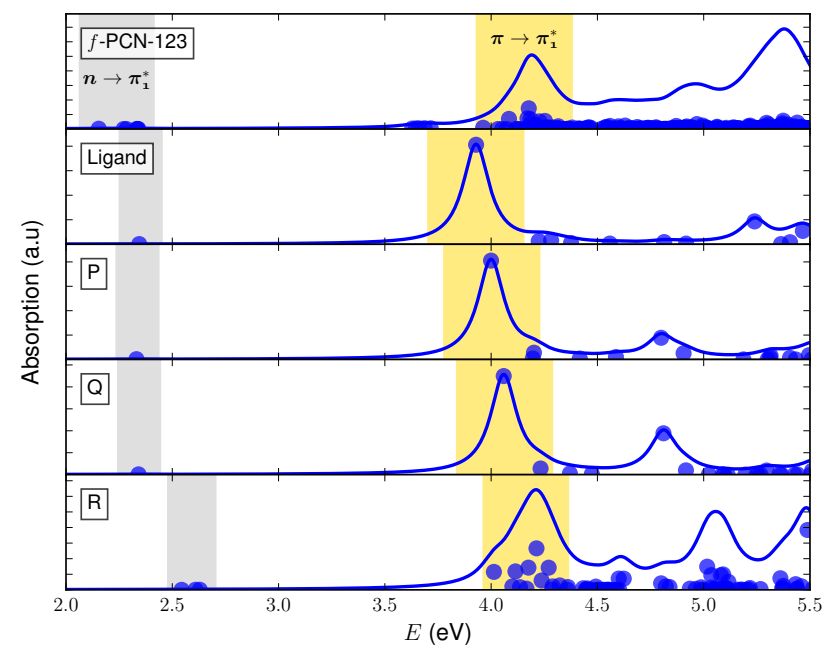

(b)

Figure 4. BSE/evGW spectra of different models of cis (a) and trans(b) PCN-123 obtained by multiplying the oscillator strength by a Lorentzian of $0.1 \mathrm{eV}$ broadening (HWHM). The color strips denote the optical bands involving excitations with similar character (see text).

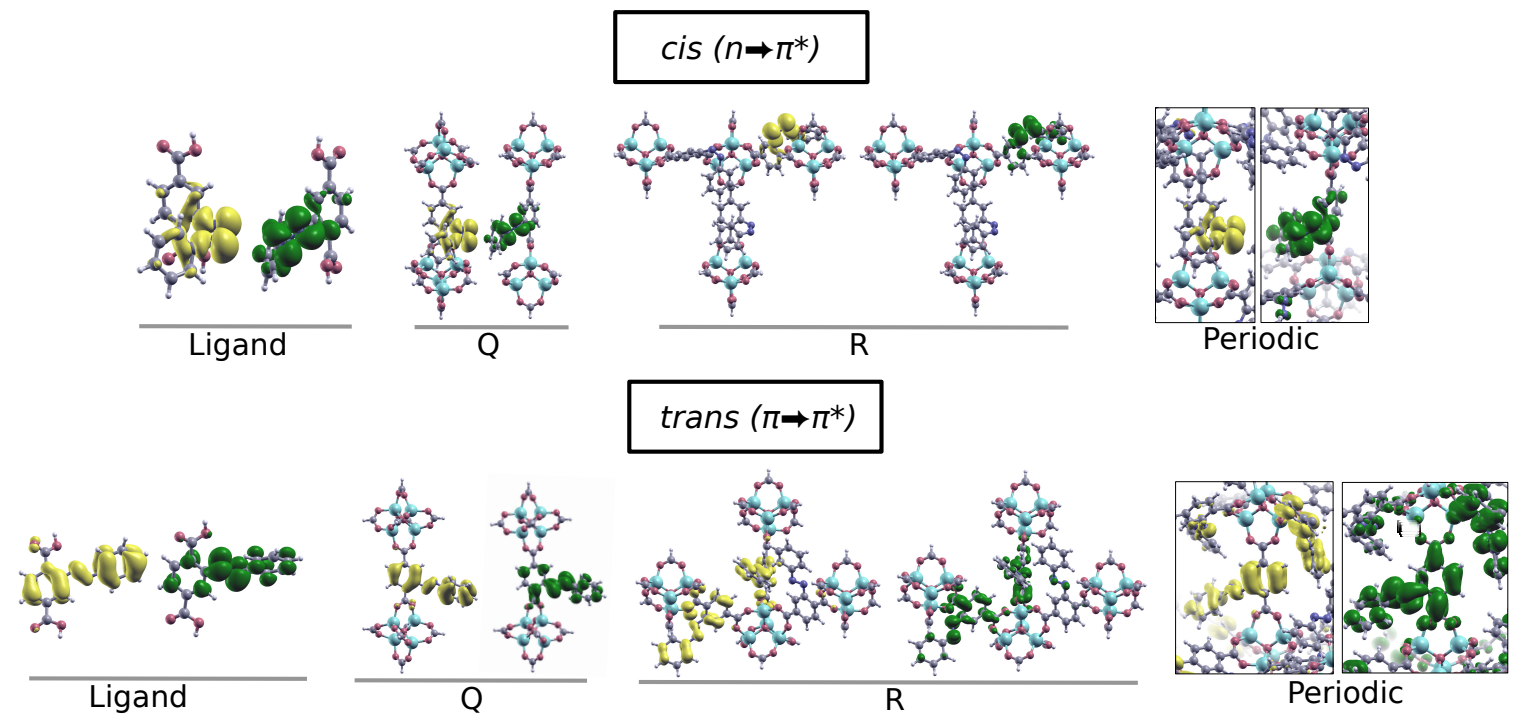

Figure 5. Excitonic densities of electron and hole as defined in eq. 4 and 5 . The densities plotted in top row correspond to $n \rightarrow \pi_{1}^{*}$ (cis) and densities in bottom row correspond to $\pi \rightarrow \pi_{1}^{*}$ (trans). The hole and electron densities are plotted in yellow and green colors, respectively.

trans and cis in the same figure, for azobenzene, the PCN123 ligand (in gas phase geometry), fragment $\mathrm{R}$ and the $f$-PCN-123 (see Fig. 6). For fragment $\mathrm{R}$ we plot also the $\mathrm{BSE} / \mathrm{evGW} / \mathrm{PBEh}$ spectra.

Moving from azobenzene to fragment $\mathrm{R}$ and finally to $f$ PCN-123, the most intense peak in both trans (i.e. $\pi \rightarrow \pi_{1}^{*}$ ) and cis (i.e. $n \rightarrow \pi_{1}^{*}$ ) undergoes a blueshift by $\sim 0.5 \mathrm{eV}$ and $\sim 0.2$, respectively. It is important to note that the $n \rightarrow \pi_{1}^{*}$ optical band of cis in the periodic calculations exhibits a significantly higher intensity compared to any other fragment model. The visual inspection of the $n \rightarrow \pi_{1}^{*}$ exciton in the different MOF models discussed above and reported in the upper panel of Fig. 5 allow to establish a good correspondence between the periodic and the fragment models. However, the spatial localization and the symmetry of the wavefunction may differ quantitatively thus affecting directly the value of the oscillator strength. As discussed above, this is confirmed by the independent-particle spectra computed for both the periodic and the fragment $\mathrm{Q}$. The two spectra have similar features and the biggest difference is the signficantly more intense first excitation in the case of $f$-PCN-123 as shown by the plotted ration between the two cases in the lower panel of Fig. S11. Thus, we attribute this discrepancy in the intensity of the first excitiation to a limitation of the fragment approach in describing the correct KS periodic eigenstates.

The excitation energies and the oscillator strengths of $n \rightarrow \pi^{*}$ excitation of azobenzene calculated with BSE/ $G W$ was shown to be very sensitive to the choice of DFT functional $^{62}$ owing to the change in the KS states. On the other hand, the position of $\pi \rightarrow \pi^{*}$ optical band is comparatively resilient to change in the DFT functional. This occurs also 

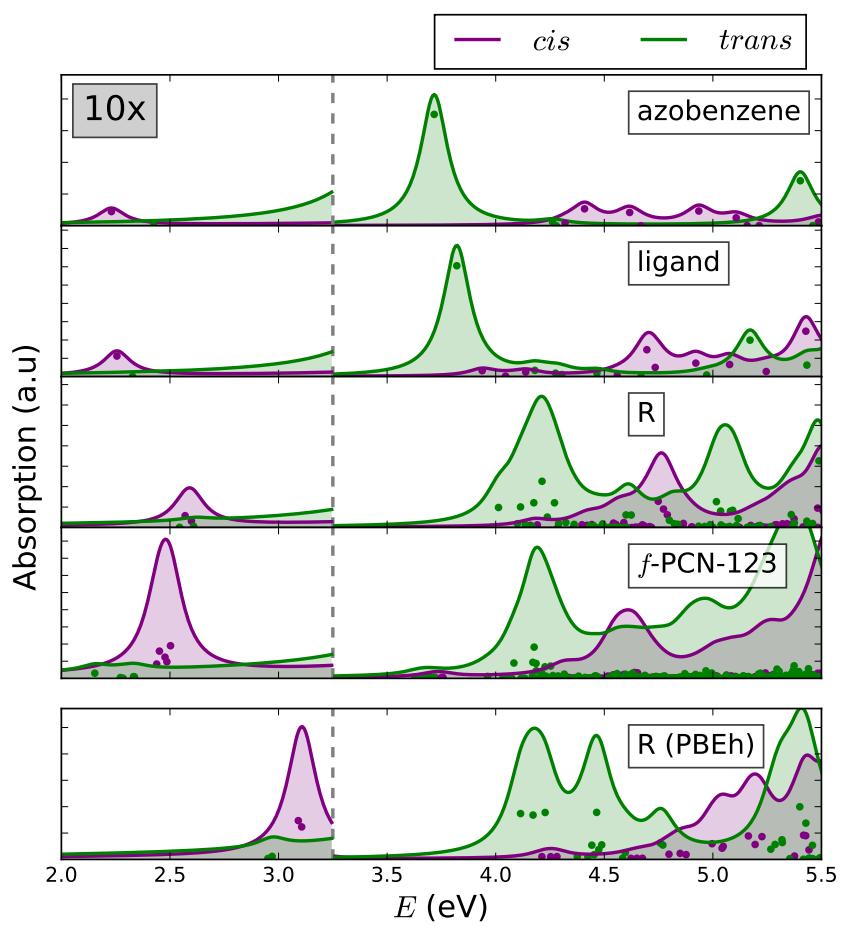

Figure 6. $\mathrm{BSE} / e v G W / \mathrm{PBE}$ spectra of azobenzene, $\mathrm{PCN}-123$ ligand, fragment $\mathrm{R}$, and $f$-PCN-123. All spectra in the low energy region are multiplied by 10 for visual clarity. The lower panel show the BSE/evGW/PBEh (40\% EXX) spectra of fragment R.

in this case: we report the comparison of the $\mathrm{BSE} / e v G W$ spectra of gas phase azobenzene computed with PBE and with the IP-tuned PBEh $(60 \%$ EXX) in Fig. S25. The experimentally-extracted values of the azobenzene $\mathrm{S}_{1}$ energy (cis: $2.92 \mathrm{eV}$, trans: $2.82 \mathrm{eV}$ ) are in excellent agreement with the BSE $\mathrm{S}_{1}$ excitation energies computed using the IP-tuned PBEh (cis: $3.00 \mathrm{eV}$, trans: $2.83 \mathrm{eV}) .{ }^{25,27,62}$ A non-negligible deviation from experiment is instead found when using the PBE starting point (cis: $2.23 \mathrm{eV}$, trans: $2.43 \mathrm{eV}$.)

In light of these results, to attempt a quantitative description of the optical properties of this material we compute the BSE spectra of fragment $\mathrm{R}$ with PBEh using $40 \%$ of exact exchange which tunes the Kohn-Sham IP to the evGW IP. The BSE spectra of either isomers is shown in the lower panel of Fig. 6. When PBEh is used, the energy of $n \rightarrow \pi_{1}^{*}$ blueshifts by $\sim 0.5 \mathrm{eV}$ for both cis and trans. The $\pi \rightarrow \pi_{1}^{*}$ excitation of trans does not vary appreciably in energy. The oscillator strength of $n \rightarrow \pi_{1}^{*}$ increases for cis from 0.012 to $0.033(\sim 2.7$ times $)$ upon change in the functional. The wide and intense band found for cis in the range of 4.5-5.0 eV is blueshifted to energies higher than $5 \mathrm{eV}$ making trans the dominant absorber between 4 and $5 \mathrm{eV}$. In case of trans, the use of hybrid functional separates the $\pi \rightarrow \pi_{1}^{*}$ excitations from $n \rightarrow \pi_{2}^{*}$ and $\pi \rightarrow \pi_{2}^{*}$ excitations resulting in two distinct optical bands instead of a single one as in the case of PBE. Like for the PBE case, the BSE/evGW/PBEh spectra show a small blueshift of the $\mathrm{S}_{1}$ of cis and the $\mathrm{S}_{2}$ of trans in the PCN-123 MOF as compared to gas phase azobenzene (in Fig. S25). The $\mathrm{S}_{1}$ of cis is at $3.0 \mathrm{eV}$ for azobenzene (see Fig. S25) and approx. $3.1 \mathrm{eV}$ for PCN-123 (see Fig. 6) and the $\mathrm{S}_{2}$ of trans at $3.9 \mathrm{eV}$ for azobenzene and 4.1-4.5 eV for PCN-123.

Finally, we briefly comment on the effect of the geometry of the azo-molecules on the optical absorption properties, in particular when fragment models are employed. Because of the structural disorder within the MOF and because the geometry of the fragments is taken by carving one (fragment $\mathrm{Q}$ ) or multiple (fragment $\mathrm{R}$ ) ligands from the periodic MOF containing six geometrically different ligands, it can happen that by taking only one of these, such as in fragment $Q$, the relative intensity between trans and cis could change. This is indeed the case when we compute the BSE/evGW/PBEh (40\% EXX) spectra of trans and cis using a fragment $Q$ taken by carving the specific ligand that exhibits the largest distortion compared to the other five ligands (and compared to gas phase). This specific choice would yield spectra with the $n \rightarrow \pi_{1}^{*}$ excitation in trans being more intense than in cis (see Fig. S26). Some caution is advised in this regard to avoid a biased result.

\section{Conclusion}

To summarize, our study of the optical absorption spectra of cis and trans PCN-123 MOF, performed by employing the $\mathrm{BSE} / \mathrm{evGW}$ approach allows to shed light for the first time on the nature of the electronic excitations of an azobenzene-functionalized MOF. Similarities and differences among the optical absorption of the fully functionalized MOF, the azobenzene molecule and the MOF azobenzenefunctionalized ligands are shown and discussed. On the computational point of view the accuracy of the fragment model is assessed by adopting a QM/MM approach where the environmental effects are considered both at the DFT level and at the $G W$ and BSE level. In the low energy region, up to $4.5 \mathrm{eV}$, a satisfactory description of the optical properties of the periodic material can be obtained already by using the smallest fragment (fragment $\mathrm{P}$ ) which consists of one metal node and a single functionalized ligand. The agreement systematically improves as we increase the size of the fragment by adding an increasing number of metal nodes and azo-functionalized ligands. In this region the spectral features of the MOF resemble closely the case of gas phase azobenzene, and the ligand. This is consistent with recent experimental studies of azobenzene functionalized IR-MOF$74,{ }^{29,30}$ ortho-fluoro-azobenzene functionalized SURMOF ${ }^{19}$ and core-shell $\mathrm{MOF}^{23}$ showing a good similarity between the optical absorption of the MOF and the ligand case. ${ }^{20}$ One important difference between the azobenzene or ligand molecule with respect to the MOF is the stronger $\mathrm{S}_{1}$ feature in cis predicted in the latter. This suggests a faster and more complete $c i s \rightarrow$ trans isomerization in the MOF upon excitation of the $S_{1}$ band, compared to the molecular case ${ }^{28,38}$ for which indeed slow and incomplete back-isomerization is observed. We believe this results has important implications for the design of photoswitches-based MOFs.

In conclusion, in terms of spectral features and character of the excitations, the periodic MOF resembles the case of free azobenzene and the azobenzene-functionalized ligand of the MOF, with the most intense bands slightly blueshifted in the MOF compared to the azobenzene molecule. Our calculations place the largest selective absorption by cis at around $3.2 \mathrm{eV}$ and by trans at $\sim 4.2 \mathrm{eV}$. The most important difference between the molecular and periodic models is the ratio between the oscillator strength of cis and trans for the $\mathrm{S}_{1}$, which is found to be significantly larger in the periodic MOF compared to any fragment model, including the free 
azobenzene molecule as discussed above. This is attributed to the limitation of non-periodic models to provide a quantitative description of the electronic structure of the periodic system at the DFT level. As the excitation energy increases the fragment model becomes less adequate to describe the MOF due to the more delocalized character of the exciton and to the increasing contribution from the metal nodes.

We hope this study will help accelerate the design of photoactive frameworks with good photoisomerization efficiencies and enhanced capture performances.

Acknowledgement This work was funded by the project ANR-15-CE06-0003-01 funded by the French National Agency for Research. Calculations were performed using resources granted by GENCI under the CINES grant number A0020907211. Additionally, the froggy platform of the CIMENT infrastructure, which is supported by the Rhone-Alpes region (GRANT CPER07_13 CIRA) and the Equip@Meso project was employed for the calculations.

\section{References}

(1) Gamonal, A.; Sun, C.; Mariano, A. L.; FernandezBartolome, E.; Guerrero-SanVicente, E.; Vlaisavljevich, B. Castells-Gil, J.; Marti-Gastaldo, C.; Poloni, R.; Wannemacher, R.; Cabanillas-Gonzalez, J.; Costa, J. S. Divergent Adsorption-Dependent Luminescence of Amino-Functionalized Lanthanide Metal-Organic Frameworks for Highly Sensitive NO2 Sensors. The Journal of Physical Chemistry Letters 2020, 11,3362-3368.

(2) Chen, Z.; Li, P.; Anderson, R.; Wang, X.; Zhang, X.; Robison, L.; Redfern, L. R.; Moribe, S.; Islamoglu, T.; GómezGualdrón, D. A.; Yildirim, T.; Stoddart, J. F.; Farha, O. K Balancing volumetric and gravimetric uptake in highly porous materials for clean energy. Science 2020, 368, 297-303.

(3) Qian, Q.; Asinger, P. A.; Lee, M. J.; Han, G.; Rodriguez, K. M.; Lin, S.; Benedetti, F. M.; Wu, A. X.; Chi, W. S.; Smith, Z. P. MOF-Based Membranes for Gas Separations. Chemical Reviews 2020, 120, 8161-8266.

(4) Yang, D.; Gates, B. C. Catalysis by Metal Organic Frameworks: Perspective and Suggestions for Future Research. ACS Catalysis 2019, 9, 1779-1798.

(5) Usman, M.; Mendiratta, S.; Lu, K.-L. Metal-Organic Frameworks: New Interlayer Dielectric Materials. ChemElectroChem 2015, 2, 786-788.

(6) Resines-Urien, E.; Burzurí, E.; Fernandez-Bartolome, E.; García García-Tuñón, M. Á.; de la Presa, P.; Poloni, R.; Teat, S. J.; Costa, J. S. A switchable iron-based coordination polymer toward reversible acetonitrile electro-optical readout. Chem. Sci. 2019, 10, 6612-6616.

(7) He, H.; Cui, Y.; Li, H.; Shao, K.; Chen, B.; Qian, G. Controllable broadband multicolour single-mode polarized laser in a dye-assembled homoepitaxial MOF microcrystal. Light: Science \&S Applications 2020, 9 .

(8) Baumann, A. E.; Burns, D. A.; Liu, B.; Thoi, V. S. Metalorganic framework functionalization and design strategies for advanced electrochemical energy storage devices. Communications Chemistry 2019, 2.

(9) Poloni, R.; Lee, K.; Berger, R. F.; Smit, B.; Neaton, J. B. Understanding Trends in CO2 Adsorption in MetalâĂŞOrganic Frameworks with Open-Metal Sites. The Journal of Physical Chemistry Letters 2014, 5, 861-865, PMID: 26274079.

(10) Poloni, R.; Kim, J. Thermodynamics of gas adsorption in MOFs using Ab Initio calculations. International Journal of Quantum Chemistry 2016, 116, 569-572.

(11) Vaidhyanathan, R.; Iremonger, S. S.; Shimizu, G. K. H. Boyd, P. G.; Alavi, S.; Woo, T. K. Direct Observation and Quantification of CO2Binding Within an Amine-Functionalized Nanoporous Solid. Science 2010, 330, 650-653.

(12) McDonald, T. M. et al. Cooperative insertion of $\mathrm{CO} 2$ in diamineappended metal-organic frameworks. Nature 2015, 519, 303308.

(13) Li, H.; Wang, K.; Sun, Y.; Lollar, C. T.; Li, J.; Zhou, H.-C. Recent advances in gas storage and separation using metalâASorganic frameworks. Materials Today 2018, 21, 108-121.

(14) Ding, M.; Flaig, R. W.; Jiang, H.-L.; Yaghi, O. M. Carbon capture and conversion using metalâĂSorganic frameworks and MOF-based materials. Chem. Soc. Rev. 2019, 48, 2783-2828.

(15) $\mathrm{Hu}, \mathrm{Z}$.; Wang, Y.; Shah, B. B.; Zhao, D. $\mathrm{CO}_{2}$ Capture in MetalOrganic Framework Adsorbents: An Engineering Perspective. Advanced Sustainable Systems 2019, 3, 1800080 .

(16) Huck, J. M.; Lin, L.-C.; Berger, A. H.; Shahrak, M. N.; Martin, R. L.; Bhown, A. S.; Haranczyk, M.; Reuter, K.; Smit, B. Evaluating different classes of porous materials for carbon cap- ture. Energy Environ. Sci. 2014, 7, 4132-4146.

(17) Park, J.; Yuan, D. Pham, K. T. Li, J-R ; Yakovenko, A Zhou, H.-C. Reversible alteration of $\mathrm{CO} 2$ adsorption upon photochemical or thermal treatment in a metal-organic framework. J. Am. Chem. Soc. 2011, 134, 99-102.

(18) Rosi, N. L.; Eckert, J.; Eddaoudi, M.; Vodak, D. T.; Kim, J.; O'Keeffe, M.; Yaghi, O. M. Hydrogen Storage in Microporous Metal-Organic Frameworks. Science 2003, 300, 1127-1129.

(19) Wang, Z.; Knebel, A.; Grosjean, S.; Wagner, D.; Bräse, S.; Wöll, C.; Caro, J.; Heinke, L. Tunable Molecular Separation by Nanoporous Membranes. Nat. Commun. 2016, 7, 13872.

(20) Müller, K.; Knebel, A.; Zhao, F.; Bléger, D.; Caro, J.; Heinke, L. Switching Thin Films of Azobenzene-Containing Metal-Organic Frameworks with Visible Light. Chem. Eur. J. 2017, 23, 54345438

(21) Kanj, A. B.; BÃijrck, J.; Grosjean, S.; BrÃd'se, S.; Heinke, L. Switching the enantioselectivity of nanoporous host materials by light. Chem. Commun. 2019, 55, 8776-8779.

(22) Jiang, Y.; Tan, P.; Qi, S.-C.; Liu, X.-Q.; Yan, J.-H.; Fan, F.; Sun, L.-B. Metal-Organic Frameworks with Target-Specific Active Sites Switched by Photoresponsive Motifs: Efficient Adsorbents for Tailorable $\mathrm{CO}_{2}$ Capture. Angewandte Chemie International Edition 2019, 58, 6600-6604.

(23) Mutruc, D.; Goulet-Hanssens, A.; Fairman, S.; Wahl, S.; Zimathies, A.; Knie, C.; Hecht, S. Modulating Guest Uptake in Core-Shell MOFs with Visible Light. Angewandte Chemie International Edition 2019, 58, 12862-12867.

(24) Yang, C.-T.; Kshirsagar, A. R.; Eddin, A. C.; Lin, L.-C. Poloni, R. Tuning Gas Adsorption by Metal Node Blocking in Photoresponsive Metal-Organic Frameworks. Chem. Eur. J. 2018, 24, 15167-15172.

(25) Andersson, J.-A.; Petterson, R.; Tegnér, L. Flash photolysis experiments in the vapour phase at elevated temperatures I: spectra of azobenzene and the kinetics of its thermal cis-trans isomerization. Journal of Photochemistry 1982, 20, 17-32.

(26) Bandara, H. M. D.; Burdette, S. C. Photoisomerization in Different Classes of Azobenzene. Chem. Soc. Rev. 2012, 41, 18091825

(27) Vetráková, L.; Ladányi, V.; Al Anshori, J.; Dvořák, P.; Wirz, J.; Heger, D. The absorption spectrum of cis-azobenzene. Photochemical \&S Photobiological Sciences 2017, 16, 1749-1756.

(28) Ladányi, V.; Dvořák, P.; Anshori, J. A.; Vetráková, L'.; Wirz, J.; Heger, D. Azobenzene photoisomerization quantum yields in methanol redetermined. Photochemical \& Photobiological Sciences 2017, 16, 1757-1761.

(29) Brown, J. W.; Henderson, B. L.; Kiesz, M. D.; Whalley, A. C.; Morris, W.; Grunder, S.; Deng, H.; Furukawa, H.; Zink, J. I.; Stoddart, J. F.; Yaghi, O. M. Photophysical pore control in an azobenzene-containing metal-organic framework. Chemical Science 2013, 4, 2858.

(30) Wang, Z.; Grosjean, S.; Bräse, S.; Heinke, L. Photoswitchable Adsorption in Metal-Organic Frameworks Based on Polar Guest-Host Interactions. ChemPhysChem 2015, 16, 37793783.

(31) Mancuso, J. L.; Mroz, A. M.; Le, K. N.; Hendon, C. H. Electronic Structure Modeling of Metal-Organic Frameworks. Chemical Reviews 2020, 120, 8641-8715.

(32) Gu, Z.-G.; Heinke, L.; Wöll, C.; Neumann, T.; Wenzel, W.; Li, Q.; Fink, K.; Gordan, O. D.; Zahn, D. R. Experimental and theoretical investigations of the electronic band structure of metal-organic frameworks of HKUST-1 type. Appl. Phys. Lett. 2015, 10\%, 1021

(33) Fumanal, M.; Corminboeuf, C.; Smit, B.; Tavernelli, I. Optical absorption properties of metal-organic frameworks: solid state versus molecular perspective. Physical Chemistry Chemical Physics 2020, 22, 19512-19521.

(34) Vermoortele, F.; Vandichel, M.; Van de Voorde, B.; Ameloot, R.; Waroquier, M.; Van Speybroeck, V.; De Vos, D. E. Electronic Effects of Linker Substitution on Lewis Acid Catalysis with Metal-Organic Frameworks. Angewandte Chemie International Edition 2012, 51, 4887-4890.

(35) Ji, M.; Lan, X.; Han, Z.; Hao, C.; Qiu, J. Luminescent Properties of Metal-Organic Framework MOF-5: Relativistic TimeDependent Density Functional Theory Investigations. Inorganic Chemistry 2012, 51, 12389-12394.

(36) Conti, I.; Garavelli, M.; Orlandi, G. The Different Photoisomerization Efficiency of Azobenzene in the Lowest $n \pi *$ and $\pi \pi *$ Singlets: The Role of a Phantom State. J. Am. Chem. Soc. 2008, 130, 5216-5230.

(37) Fliegl, H.; Köhn, A.; Hättig, C.; Ahlrichs, R. Ab Initio Calculation of the Vibrational and Electronic Spectra of trans-and cis-Azobenzene. J. Am. Chem. Soc. 2003, 125, 9821-9827.

(38) Zimmerman, G.; Chow, L.-Y.; Paik, U.-J. The Photochemical Isomerization of Azobenzene. J. Am. Chem. Soc. 1958, 80 , $3528-3531$

(39) Duarte, L.; Fausto, R.; Reva, I. Structural and spectroscopic characterization of E-and Z-isomers of azobenzene. Phys. Chem. Chem. Phys. 2014, 16, 16919-16930.

(40) Crecca, C. R.; Roitberg, A. E. Theoretical study of the isomerization mechanism of azobenzene and disubstituted azobenzene derivatives. J. Phys. Chem. A 2006, 110, 8188-8203.

(41) Salpeter, E. E.; Bethe, H. A. A Relativistic Equation for BoundState Problems. Physical Review 1951, 84, 1232-1242.

(42) Albrecht, S.; Reining, L.; Sole, R. D.; Onida, G. Ab InitioCal- 
culation of Excitonic Effects in the Optical Spectra of Semiconductors. Physical Review Letters 1998, 80, 4510-4513.

(43) Strinati, G. Application of the Green's functions method to the study of the optical properties of semiconductors. La Rivista del Nuovo Cimento 1988, 11, 1-86.

(44) Martin, R. M.; Reining, L.; Ceperley, D. M. Interacting Electrons; Cambridge University Press, 2016.

(45) Rohlfing, M.; Louie, S. G. Electron-Hole Excitations in Semiconductors and Insulators. Physical Review Letters 1998, 81, $2312-2315$

(46) Benedict, L. X.; Shirley, E. L.; Bohn, R. B. Optical Absorption of Insulators and the Electron-Hole Interaction: AnAb InitioCalculation. Physical Review Letters 1998, 80, 4514-4517.

(47) Onida, G.; Reining, L.; Rubio, A. Electronic excitations density-functional versus many-body Green's-function approaches. Reviews of Modern Physics 2002, 74, 601-659.

(48) Palummo, M.; Hogan, C.; Sottile, F.; BagalÃa, P.; Rubio, A. Ab initio electronic and optical spectra of free-base porphyrins: The role of electronic correlation. The Journal of Chemical Physics 2009, 131, 084102.

49) Ping, Y.; Rocca, D.; Galli, G. Electronic excitations in light absorbers for photoelectrochemical energy conversion: first principles calculations based on many body perturbation theory. Chemical Society Reviews 2013, 42, 2437.

(50) Blase, X.; Duchemin, I.; Jacquemin, D. The Bethe-Salpeter Equation in Chemistry: Relations with TD-DFT, Applications and Challenges. Chem. Soc. Rev, 2018, 47, 1022-1043.

(51) Hedin, L. New Method for Calculating the One-Particle Green's Function with Application to the Electron-Gas Problem. Physical Review 1965, 139, A796-A823.

(52) Strinati, G.; Mattausch, H. J.; Hanke, W. Dynamical Correlation Effects on the Quasiparticle Bloch States of a Covalent Crystal. Physical Review Letters 1980, 45, 290-294.

(53) Hybertsen, M. S.; Louie, S. G. Electron correlation in semiconductors and insulators: Band gaps and quasiparticle energies. Physical Review B 1986, 34, 5390-5413.

(54) Casida, M. E. Time-dependent density-functional theory for molecules and molecular solids. Journal of Molecular Structure: THEOCHEM 2009, 914, 3-18.

(55) Faber, C.; Boulanger, P.; Attaccalite, C.; Duchemin, I.; Blase, X. Excited states properties of organic molecules: from density functional theory to the GW and Bethe-Salpeter Green's function formalisms. Phil. Trans. R. Soc. A 2014, 372, 20130271

(56) Jacquemin, D.; Duchemin, I.; Blase, X. Is the Bethe-Salpeter Formalism Accurate for Excitation Energies? Comparisons with TD-DFT, CASPT2, and EOM-CCSD. The Journal of Physical Chemistry Letters 2017, 8, 1524-1529.

(57) Jacquemin, D.; Duchemin, I.; Blase, X. Benchmarking the Bethe-Salpeter Formalism on a Standard Organic Molecular Set. Journal of Chemical Theory and Computation 2015, 11, 3290-3304.

(58) Bruneval, F.; Hamed, S. M.; Neaton, J. B. A systematic benchmark of the ab initio Bethe-Salpeter equation approach for lowlying optical excitations of small organic molecules. The Journal of Chemical Physics 2015, 142, 244101.

(59) Perdew, J. P. Burke, K.; Ernzerhof, M. Generalized Gradient Approximation Made Simple. Phys. Rev. Lett. 1996, 77, 38653868

(60) Perdew, J. P.; Ernzerhof, M.; Burke, K. Rationale for mixing exact exchange with density functional approximations. The Journal of Chemical Physics 1996, 105, 9982-9985.

(61) Perdew, J. P.; Levy, M. Comment on "Significance of the Highest Occupied Kohn-Sham Eigenvalue". Phys. Rev. B 1997, 56 , $16021-16028$

(62) Kshirsagar, A. R.; D'Avino, G.; Blase, X.; Li, J.; Poloni, R Accurate Prediction of the S1 Excitation Energy in Solvated Azobenzene Derivatives via Embedded Orbital-Tuned BetheSalpeter Calculations. Journal of Chemical Theory and Computation 2020, 16, 2021-2027.

(63) Blase, X.; Attaccalite, C.; Olevano, V. First-Principles $G W$ calculations for fullerenes, porphyrins, phtalocyanine, and other Molecules of Interest for Organic Photovoltaic Applications. Phys. Rev. B 2011, 83, 115103.

(64) Kaplan, F.; Harding, M. E.; Seiler, C.; Weigend, F.; Evers, F.; van Setten, M. J. Quasi-Particle Self-Consistent GW for Molecules. J. Chem. Theory Comput. 2016, 12, 2528-2541.

(65) Giannozzi, P. et al. QUANTUM ESPRESSO: a modular and open-source software project for quantum simulations of materials. Journal of Physics: Condensed Matter 2009, 21, 395502

(66) Giannozzi, P. et al. Advanced capabilities for materials modelling with Quantum ESPRESSO. Journal of Physics: Condensed Matter 2017, 29, 465901.

(67) van Setten, M.; Giantomassi, M.; Bousquet, E.; Verstraete, M. Hamann, D.; Gonze, X.; Rignanese, G.-M. The PseudoDojo: Training and grading a 85 element optimized norm-conserving pseudopotential table. Computer Physics Communications 2018, 226, 39-54.

(68) Hamann, D. R. Optimized norm-conserving Vanderbilt pseudopotentials. Physical Review B 2013, 88 .

(69) Hamada, I. van der Waals density functional made accurate. Physical Review $B$ 2014, 89.

(70) Marini, A.; Hogan, C.; Grüning, M.; Varsano, D. yambo: An ab initio tool for excited state calculations. Computer Physics
Communications 2009, 180, 1392-1403.

71) Sangalli, D et al. Many-body perturbation theory calculation using the yambo code. Journal of Physics: Condensed Matter 2019, 31, 325902

(72) Oschlies, A.; Godby, R. W.; Needs, R. J. GWself-energy calculations of carrier-induced band-gap narrowing inn-type silicon. Physical Review B 1995, 51, 1527-1535.

(73) Bruneval, F.; Gonze, X. AccurateGWself-energies in a planewave basis using only a few empty states: Towards large systems. Physical Review B 2008, 78

(74) Aprà, E. et al. NWChem: Past, present, and future. The Journal of Chemical Physics 2020, 152, 184102.

(75) Dunning Jr, T. H. Gaussian Basis Sets for Use in Correlated Molecular Calculations. I. The Atoms Boron through Neon and Hydrogen. J. Chem. Phys. 1989, 90, 1007-1023.

(76) Woon, D. E.; Dunning Jr, T. H. Gaussian basis sets for use in correlated molecular calculations. III. The atoms aluminum through argon. J. Chem. Phys. 1993, 98, 1358-1371.

(77) Papajak, E.; Leverentz, H. R.; Zheng, J.; Truhlar, D. G. Efficient Diffuse Basis Sets: cc-pVxZ+ and maug-cc-pVxZ. Journal of Chemical Theory and Computation 2009, 5, 1197-1202.

(78) Roy, L. E.; Hay, P. J.; Martin, R. L. Revised basis sets for the LANL effective core potentials. J. Chem. Theory Comput. 2008, 4, 1029-1031.

(79) Hay, P. J.; Wadt, W. R. Ab initio effective core potentials for molecular calculations. Potentials for the transition metal atoms Sc to Hg. The Journal of Chemical Physics 1985, 82, 270-283.

(80) Blase, X. Attaccalite, C. Charge-Transfer Excitations in Molecular Donor-Acceptor Complexes within the Many-Body BetheSalpeter Approach. Appl. Phys. Lett. 2011, 99, 171909

(81) Weigend, F. Accurate Coulomb-fitting basis sets for $\mathrm{H}$ to $\mathrm{Rn}$ Physical Chemistry Chemical Physics 2006, 8, 1057.

(82) York, D. M.; Karplus, M. A Smooth Solvation Potential based on the Conductor-Like Screening Model. J. Phys. Chem. A 1999, 103, 11060-11079.

(83) Duchemin, I.; Jacquemin, D.; Blase, X. Combining the GW formalism with the polarizable continuum model: A state-specific non-equilibrium approach. $J$. Chem. Phys, 2016, 144, 164106.

(84) Li, J.; D’Avino, G.; Duchemin, I.; Beljonne, D.; Blase, X. Combining the Many-Body GW Formalism with Classical Polarizable Models: Insights on the Electronic Structure of Molecular Solids. J. Phys. Chem. Lett. 2016, 7, 2814-2820.

(85) D'Avino, G.; Muccioli, L.; Zannoni, C.; Beljonne, D.; Soos, Z. G. Electronic Polarization in Organic Crystals: A Comparative Study of Induced Dipoles and Intramolecular Charge Redistribution Schemes. J. Chem. Theory Comput. 2014, 10, 4959 4971

(86) Li, J.; D’Avino, G.; Duchemin, I.; Beljonne, D.; Blase, X. Accurate description of charged excitations in molecular solids from embedded many-body perturbation theory. Phys. Rev. B 2018 97, 035108

(87) Duchemin, I.; Guido, C. A.; Jacquemin, D.; Blase, X. The Bethe-Salpeter formalism with polarisable continuum embedding: reconciling linear-response and state-specific features. Chem. Sci. 2018, 9, 4430-4443.

(88) van Setten, M. J.; Caruso, F.; Sharifzadeh, S.; Ren, X.; Scheffler, M.; Liu, F.; Lischner, J.; Lin, L.; Deslippe, J. R.; Louie, S. G.; Yang, C.; Weigend, F.; Neaton, J. B.; Evers, F.; Rinke, P. GW100: Benchmarking G0W0 for Molecular Systems. Journal of Chemical Theory and Computation 2015, 11, 56655687, PMID: 26642984.

(89) Golze, D.; Dvorak, M.; Rinke, P. The GW Compendium: A Practical Guide to Theoretical Photoemission Spectroscopy. Frontiers in Chemistry 2019, 7.

(90) Rangel, T.; Hamed, S. M.; Bruneval, F.; Neaton, J. B. Evaluating the GW Approximation with $\operatorname{CCSD}(\mathrm{T})$ for Charged Excitations Across the Oligoacenes. Journal of Chemical Theory and Computation 2016, 12, 2834-2842.

(91) Marom, N.; Caruso, F.; Ren, X.; Hofmann, O. T.; Körzdörfer, T.; Chelikowsky, J. R.; Rubio, A.; Scheffler, M.; Rinke, P. Benchmark ofGWmethods for azabenzenes. Physical Review $B$ 2012, 86 .

(92) Bruneval, F.; Marques, M. A. L. Benchmarking the Starting Points of the GW Approximation for Molecules. Journal of Chemical Theory and Computation 2012, 9, 324-329.

(93) Faber, C. Electronic, excitonic and polaronic properties of organic systems within the many-body GW and Bethe-Salpeter formalisms : towards organic photovoltaics. Theses, Université de Grenoble, 2014.

(94) Beharry, A. A.; Sadovski, O.; Woolley, G. A. Azobenzene Photoswitching without Ultraviolet Light. Journal of the American Chemical Society 2011, 133, 19684-19687. 